\title{
Optical detection of acetylcholine esterase based on CdTe quantum dots
}



\author{
CHEN ZhenZhen, REN XiangLing \& TANG FangQiong* \\ Laboratory of Controllable Preparation and Application of Nanomaterials, Technical Institute of Physics and Chemistry, Chinese Academy of \\ Sciences, Beijing 100190, China
}

Received July 13, 2012; accepted September 3, 2012; published online January 4, 2013

\begin{abstract}
In this study, we have constructed a simple, sensitive and rapid biosensor for detection of acetylcholine esterase (AChE) based on CdTe quantum dots (QDs). The detection limit of AChE by one-step enzyme reaction based on the thiolglycolic acid (TGA) stabilized QDs (TGA-QDs) was $10 \mathrm{U} / \mathrm{L}$ and the linear range was 10-100 and 100-1200 U/L, respectively. The detection limit of AChE by two-step enzyme reaction based on the 3-mercaptopropionic acid (MGA) stabilized QDs (MGA-QDs) was found to be $20 \mathrm{U} / \mathrm{L}$ and the linear range was 100-2500 U/L. The experimental conditions of biosensors were optimized, and the detection mechanism was studied. We also detected AChE in serum samples based on TGA-QDs or MGA-QDs. The linear range was 10-140 and 50-1000 U/L, respectively. The excellent performance of this novel biosensor demonstrated that this strategy has prodigious potential to be applied in practice detection of $\mathrm{AChE}$.
\end{abstract}

quantum dots, fluorescence, detection, acetylcholine esterase

Citation: $\quad$ Chen Z Z, Ren X L, Tang F Q. Optical detection of acetylcholine esterase based on CdTe quantum dots. Chin Sci Bull, 2013, 58: 2622-2627, doi: $10.1007 / \mathrm{s} 11434-012-5590-4$

CdTe quantum dots (QDs) is a kind of fluorescent nanoparticles of intense research and broad applications [1-7]. Comparing to fluorescent proteins and organic dyes, QDs have unique structural and functional properties, such as composition and size tunable fluorescence emission, exceptional brightness and photostability, and large absorption cross sections. In recent years, a lot of researches focus on QDs-enzyme biosensors [8] due to their novel, unique properties and broad biomedical applications. However, these researches mainly focus on some routine substance such as glucose [9-12]. There have been no studies on the detection of AChE based on QDs which also perform vital roles in human body. The decrease of AChE makes individuals prone to various nerve disorders including Alzheimer's disease, Parkinson's disease, and multiple sclerosis [13]. Therefore, the quantitative determination of AChE is very important in clinical analysis (such as human serum,

*Corresponding author (email: tangfq@mail.ipc.ac.cn) amniotic fluid, brain extracts, bile, and pharmaceutical products) and other fields. Up to date, many different strategies have been applied in the quantitative determination of AChE. However, these detection methods are still facing some problems. The electrochemical detection platforms required complicated modifications of the electrodes and the HPLC detection systems needed complex operation and long time, etc. Therefore it urgently needs to develop a precise, simple, sensitive, and convenient detection strategy for AChE.

In this paper, we have constructed a simple, sensitive and rapid AChE measuring strategy based on the CdTe QDs. The biosensor was composed of QDs [thiolglycolic acid (TGA) stabilized QDs (TGA-QDs) or 3-mercaptopropionic acid (MGA) stabilized QDs (MGA-QDs)] and enzyme, without any further complex procedures of functionalization and conjugation. This convenient detection of $\mathrm{AChE}$ was based on the fluorescence enhancement or fluorescence quenching of the QDs by $\mathrm{H}_{2} \mathrm{O}_{2}$ or acetic acid which was 
produced from the enzymatic reaction of AChE. The fabricated optical biosensor platform showed fast analysis, wide linear detection range, low detection limit and good selectivity. This optical analysis strategy could provide a generic way to analyze numerous $\mathrm{H}_{2} \mathrm{O}_{2}$-dependent or acetic aciddependent enzymes because a lot of enzymatic reactions could produce hydrogen peroxide or acetic acid.

More important is AChE detected by one-step enzyme reaction for the first time in this paper. The decomposition of ACh usually requires two-step enzyme reaction. In the first step, there is not any chromophore or electroactive species generated which makes it hard to apply to optical or electrochemical detection. The previous study of AChE detection was most based on the $\mathrm{H}_{2} \mathrm{O}_{2}$ generated in the second step enzyme reaction. In this paper, we found that TGA-QDs had a significant response of acetic acids generated in the first step enzyme reaction. So we can detect AChE only based on the one-step enzyme reaction. The one enzyme detection was simpler than two-step enzyme reaction, and can reduce costs and decrease the measuring error introduced by multiple enzyme catalyzed reaction. This will help the construction of analysis device and make the detection signal more stable.

\section{Materials and methods}

\subsection{Materials}

Choline oxidase (ChOx), acetylcholine chloride (ACh) and acetylcholine esterase (AChE) were obtained from Sigma. All reagents were analytical grade and used as received.

\subsection{Synthesis of TGA-QDs and MGA-QDs}

The TGA-QDs were fabricated via a strategy described in a previous literature with minor modification [14]. Briefly, the CdTe precursor solution was prepared by adding freshly prepared $\mathrm{NaHTe}$ solution to a nitrogen-saturated $\mathrm{Cd}\left(\mathrm{NO}_{3}\right)_{2}$ solution at $\mathrm{pH} 10$ in the presence of thiolglycolic acid (TGA) as stabilizer. The concentrations of the precursor were $[\mathrm{Cd}]$ $=10 \mathrm{mmol} / \mathrm{L},[\mathrm{TGA}]=14 \mathrm{mmol} / \mathrm{L},[\mathrm{Te}]=5 \mathrm{mmol} / \mathrm{L}$, respectively. The CdTe precursor solution was heated at $90^{\circ} \mathrm{C}$ for $9 \mathrm{~h}$. The synthesis operation of MGA-QDs was almost the same as TGA-QDs. The only difference was the replacement of TGA by MGA.

\subsection{Instrumentation}

Cary Eclipse fluorescence spectrophotometer (Varian, Inc.) was employed for fluorescence measurements. The emission spectra were recorded in the wavelength of 500-680 $\mathrm{nm}$ upon excitation at $480 \mathrm{~nm}$. The exciting slit and the emission slit were 5 and $5 \mathrm{~nm}$, respectively. The samples for the fluorescence measurements were placed in a $10 \mathrm{~mm}$ optical path length quartz fluorescence cuvette.

\subsection{Fluorescence experiments}

The AChE detection procedure based on TGA-QDs is below: $20 \mu \mathrm{L}$ of TGA-QDs was diluted into $400 \mu \mathrm{L}$, and then reacted with $0.5 \mathrm{mmol} / \mathrm{L}$ or $1 \mathrm{mmol} / \mathrm{L} \mathrm{ACh}$ and different concentrations of AChE solution (concentration of 10-140 or 100-1200 U/L) for $10 \mathrm{~min}$.

The AChE detection procedure based on MGA-QDs is: $10 \mu \mathrm{L}$ of MGA-QDs was diluted into $400 \mu \mathrm{L}$, and then reacted with $1250 \mathrm{U} / \mathrm{L}$ ChOx, $200 \mu \mathrm{mol} / \mathrm{L} \mathrm{ACh}$ and different concentrations of AChE solution (concentration from 20 to $3000 \mathrm{U} / \mathrm{L}$ ) for $10 \mathrm{~min}$.

\section{Results and discussion}

\subsection{Fluorescence detection of AChE based on one-step enzyme reaction}

AChE is a serine protease that hydrolyzes the neurotransmitter ACh. AChE is mainly found at neuromuscular junctions and cholinergic brain synapses, where its activity serves to terminate synaptic transmission. It belongs to carboxylesterase family of enzymes. In the biochemical reaction, the AChE catalyzes ACh with the generation of choline (Reaction 1), and $\mathrm{ChOx}$ can catalyze the choline to generate $\mathrm{H}_{2} \mathrm{O}_{2}$ (Reaction 2). The biochemical reaction of the ACh occurs as the following:



In the first step, there is no any chromophore or electroactive species generated which makes it hard to apply to optical or electrochemical detection. The previous study of AChE detection was most based on the $\mathrm{H}_{2} \mathrm{O}_{2}$ generated in the second step enzyme reaction. In this paper, we found that TGA-QDs had a significant response of acetic acids generated in the first step enzyme reaction. So, we can detect $\mathrm{AChE}$ based on the first step enzyme reaction. Here, the fluorescence intensities of the TGA-QDs affected by acetic acid were measured. Figure 1A shows the fluorescence changes of the TGA-QDs mixing with different concentrations of acetic acid. As shown in Figure 1A, the acetic acid can effectively enhance the fluorescence intensity of TGA-QDs. So based on the fluorescence enhancement of TGA-QDs caused by acetic acid, we can realize the detection of AChE.

Figure 1B shows the fluorescence enhancement of TGAQDs upon interaction with $0.5 \mathrm{mmol} / \mathrm{L} \mathrm{ACh}$ and different concentrations of ACh. As the concentration of AChE increased, the fluorescence of the TGA-QDs was enhanced. Calibration curve in the inset was the plots of the optical analysis of different concentrations of AChE by the fluorescence maximum of the TGA-QDs. The results exhibited 

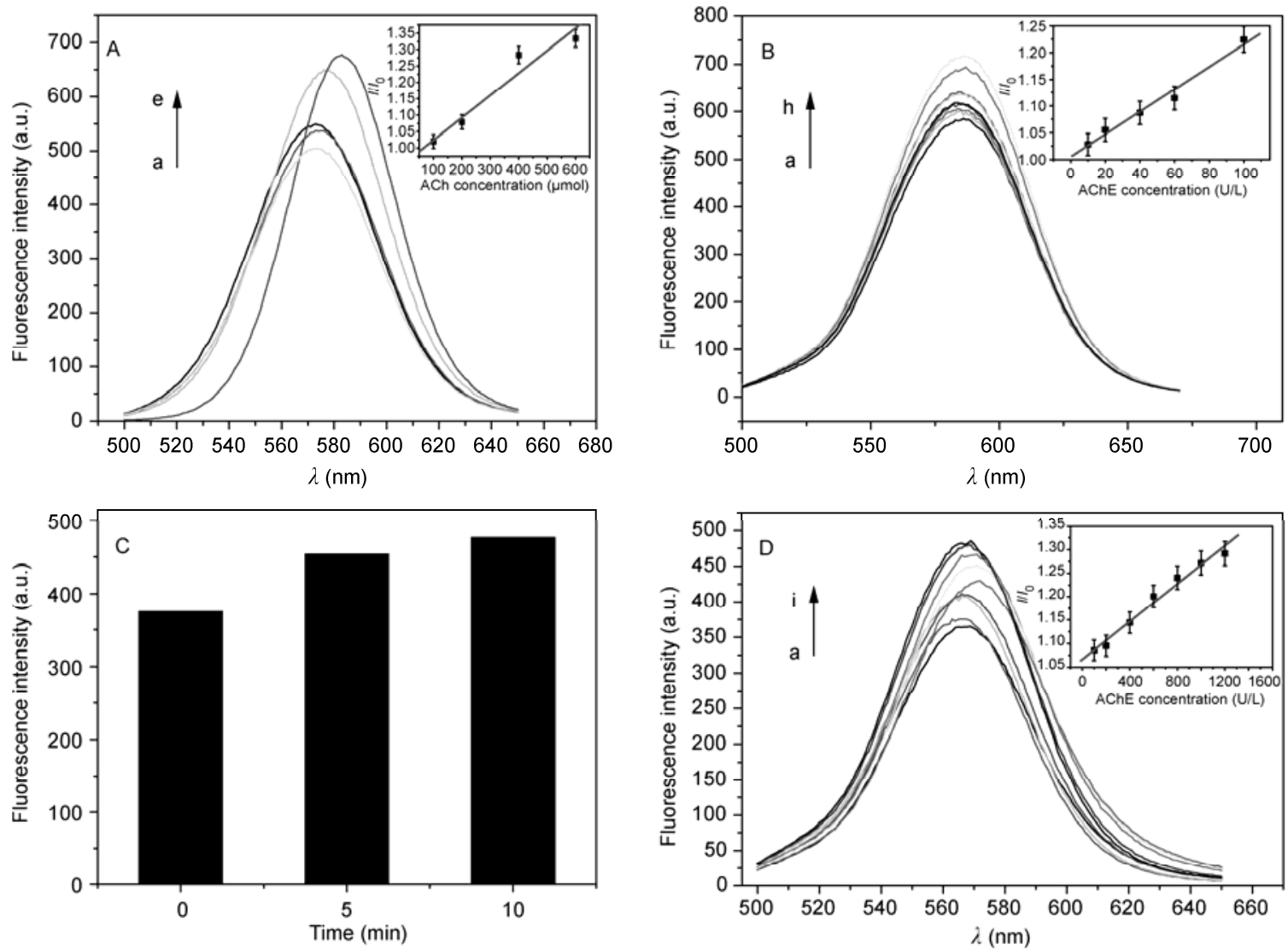

Figure 1 (A) Fluorescence changes upon the interaction of TGA-QDs with different concentrations of acetic acid. a-e: 0, 100, 200, 400, 600 $\mu \mathrm{mol} / \mathrm{L}$, respectively. (B) Fluorescence spectra of TGA-QDs in the presence of $0.5 \mathrm{mmol} / \mathrm{L}$ ACh and varying concentrations of AChE. a-h: 0, 10, 20, 40, 60, 100, 120, $140 \mathrm{U} / \mathrm{L}$. The inset displays plots of the relative fluorescence intensity of TGA-QDs versus the concentration of AChE. (C) Time-dependent fluorescence changes of TGA-QDs in the presence of $1 \mathrm{mmol} / \mathrm{L}$ ACh and $1000 \mathrm{U} / \mathrm{L}$ AChE: $0,5,10 \mathrm{~min}$. (D) Fluorescence spectra of TGA-QDs in the presence of $1 \mathrm{mmol} / \mathrm{L}$ ACh and varying concentrations of AChE. a-j: 0, 100, 200, 400, 600, 800, 1000, 1200, $1400 \mathrm{U} / \mathrm{L}$. The inset displays plots of the relative fluorescence intensity of TGA-QDs versus the concentration of AChE.

a good linear relationship in the range of $10-100 \mathrm{U} / \mathrm{L}$. The limit of AChE detection was $10 \mathrm{U} / \mathrm{L}$. Figure $1 \mathrm{C}$ shows the time-dependent fluorescence enhancement of the TGA-QDs upon treatment of the TGA-QDs with $1 \mathrm{mmol} / \mathrm{L}$ ACh and $1000 \mathrm{U} / \mathrm{L} \mathrm{AChE}$. As the time was prolonged, the fluorescence intensity of TGA-QDs was enhanced. This result indicated that the fluorescence intensity of TGA-QDs had been improved by acetic acid generated during the AChE catalytic reaction. The linear relationship was in high concentration (100-1200 U/L, shown in Figure 1D) when we added $1 \mathrm{mmol} / \mathrm{L}$ ACh in the detection system. The results proved that the developed TGA-QDs biosensor exhibited a rather high sensitivity and proper detection range which can realize the detection of $\mathrm{AChE}$ both in low and high concentrations. Surprisingly, the fluorescence intensity of MGAQDs did not show regular changes when different concentrations of $\mathrm{AChE}$ were added in the one-step enzyme reaction system (Figure S1). It may be because those MGA-QDs have a longer carbon chain than TGA-QDs, which makes the MGA-QDs more stable. So, the TGA-QDs were more suitable for the single-enzyme detection system.

The fluorescence of TGA-QDs changed slightly in the presence of $\mathrm{AChE}$ and $\mathrm{ACh}$ respectively (Figure S2). So, the fluorescence enhancement was caused by the enzyme reaction product-acetic acid. The possible schematic illustration of AChE biosensor based on acetic TGA-QDs was shown in Scheme 1. During the detection of AChE based on TGA-QDs, AChE diffused to the surface of TGA-QDs and catalyzed ACh to produce acetic acid (Reaction (1)), and then the acetic acid caused fluorescence enhancement of TGA-QDs. By the analysis of the change regularity of fluorescence intensity, we can realize the AChE detection.

\subsection{Fluorescence detection of AChE based on two-step enzyme reaction}

In our previous study, MGA-QDs were successfully used to detect choline and ACh based on the fluorescence quenching of MGA-QDs by $\mathrm{H}_{2} \mathrm{O}_{2}$ [15]. The enzyme reaction of AChE and ChOx generates $\mathrm{H}_{2} \mathrm{O}_{2}$ as a product shown in Reaction (2). Thus, controlling the properties of MGA-QDs quenched by $\mathrm{H}_{2} \mathrm{O}_{2}$ can realize the detection of $\mathrm{AChE}$.

We have experimented for the time effect on detection system based on MGA-QDs. The fluorescence changes in 


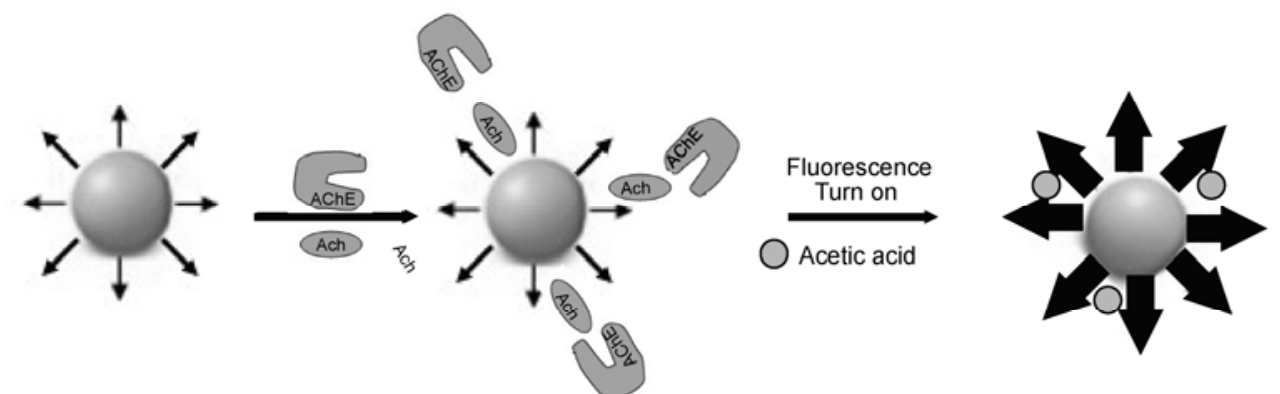

Scheme 1 Schematic principle for assay of AChE by TGA-QDs.

the presence of $1250 \mathrm{U} / \mathrm{L} \mathrm{ChOx,} 200 \mu \mathrm{mol} / \mathrm{L} \mathrm{ACh}$ and 1000 $\mathrm{U} / \mathrm{L}$ AChE for a fixed time interval of $10 \mathrm{~min}$ are shown in Figure 2A. From Figure 2A, we can see that the fluorescence maximum of MGA-QDs was observed to be quenched.

Figure 2B shows the degree of fluorescence quenching effect of MGA-QDs upon interaction with different concentrations of AChE for a fixed time interval of $10 \mathrm{~min}$. As the concentration of AChE increased, the quenching effect of the MGA-QDs was enhanced. The calibration curve of the different concentrations of AChE is shown in the inset of Figure $2 \mathrm{~B}$. The plots exhibited a good linear relationship of $100-2500 \mathrm{U} / \mathrm{L}$.

Control experiments of $\mathrm{AChE}$ detection system based on



MGA-QDs show that the fluorescence intensity of MGAQDs changed slightly in the presence of $\mathrm{ACh}, \mathrm{ChOx}$ and AChE respectively (Figure $\mathrm{S} 3$ ). The results proved that the formations of $\mathrm{H}_{2} \mathrm{O}_{2}$ are essential to quenching the fluorescence maximum of the MGA-QDs. The proposed mechanism is shown in Scheme 2. During the AChE detection by MGA-QDs, ACh was catalyzed by AChE and $\mathrm{ChOx}$ to produce $\mathrm{H}_{2} \mathrm{O}_{2}$. Then $\mathrm{H}_{2} \mathrm{O}_{2}$ diffused to the surface of MGAQDs and made electron transfer (ET) process with MGAQDs. The fluorescence of the MGA-QDs was quenched. With the amount of AChE increasing, the quenching effect of the MGA-QDs has been enhanced, because the produced $\mathrm{H}_{2} \mathrm{O}_{2}$ was increased. So the AChE can be detected based on this principle.

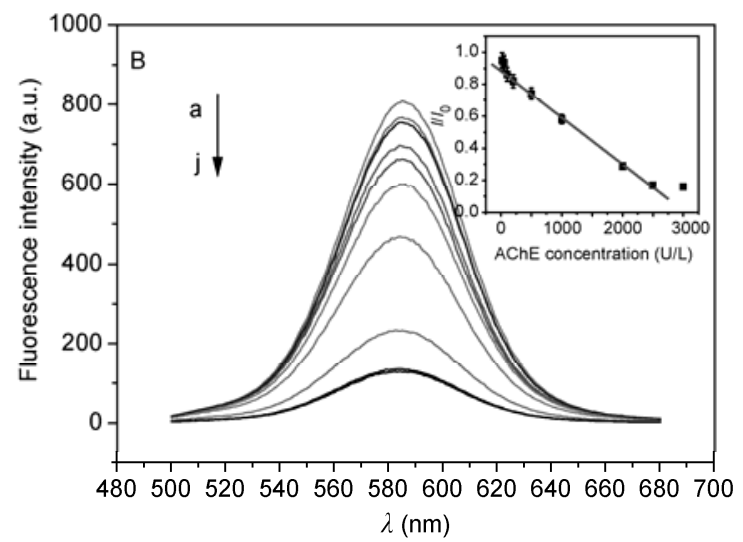

Figure 2 (A) Time-dependent fluorescence changes of MGA-QDs in the presence of $1250 \mathrm{U} / \mathrm{L}$ ChOx, $200 \mu \mathrm{mol} / \mathrm{L}$ ACh and 1000 U/L AChE. (B) Fluorescence spectra of MGA-QDs in the presence of $1250 \mathrm{U} / \mathrm{L} \mathrm{ChOx}, 200 \mu \mathrm{mol} / \mathrm{L}$ ACh and varying concentrations of ACh. a-j: 0, 20, 50, 100, 200, 500, 1000, 2000, 2500, $3000 \mathrm{U} / \mathrm{L}$. The inset displays plots of the relative fluorescence intensity of MGA-QDs versus the concentration of AChE.
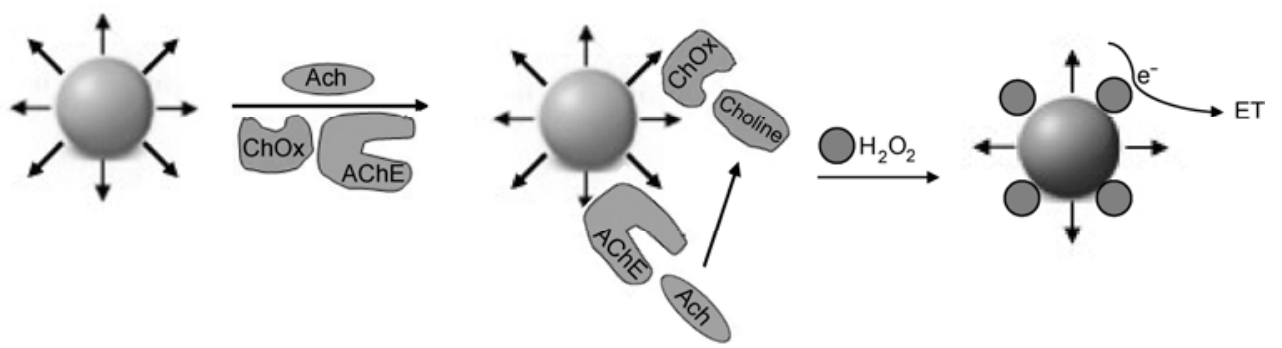

Scheme 2 Schematic principle for assay of AChE by MGA-QDs. 


\subsection{The preliminary study on application}

The AChE detection in serum was very important for the application of detection method. However, the determination of AChE in serum still existed some problems, such as easily interfered, required complicated manipulation and expensive instrumentation. Hence, there urgently needs a simple and accurate method to detect AChE in serum sample. The TGA-QDs and MGA-QDs biosensor in this paper had been tested to detect AChE in serum and proved to be a feasible method.

To the TGA-QDs biosensor, the solution samples were mixed with TGA-QDs, serum, $0.5 \mathrm{mmol} / \mathrm{L} \mathrm{ACh}$ and different concentrations of AChE. As shown in Figure 3A, the fluorescence intensity of the TGA-QDs was gradually enhanced with the increasing of AChE. The linear plot shows that the linear calibration range was 10-140 U/L (see inset in Figure 3A). To the MGA-QDs biosensor, the solution samples were mixed with MGA-QDs, serum, $1250 \mathrm{U} / \mathrm{L}$ ChOx, $200 \mu \mathrm{mol} / \mathrm{L} \mathrm{ACh}$ and different concentrations of AChE. As shown in Figure 3B, the fluorescence intensity of the MGA-QDs was gradually quenched with the increasing of AChE. The linear plot shows that the linear calibration range was 50-1000 U/L (see inset in Figure 3B). Moreover, the recovery of the AChE in serum is shown in Tables 1 and 2 . From the tables, we can see clearly that these data determined by our fabricated AChE biosensor have a good agreement with the added concentration of $\mathrm{AChE}$ in the serum sample. It proved that the AChE biosensor has a great potential for the practical biomedical applications.

\section{Conclusions}

In this paper, the TGA-QDs and MGA-QDs have been successfully employed to develop new fluorescent biosensors for selective and sensitive detection of AChE, respectively. This strategy provides a new simple and feasible way to detect AChE combining the advantages of QDs and specificity in enzymic catalytic reaction. The biosensor showed relatively high sensitivity, rapid response, low detection limit, broad linear range and good reproducibility. This strategy can be widely applied in pharmaceuticals, clinical analysis and so on. In addition, the detection limit based on one-step enzyme reaction was lower than that of two-step enzyme reaction. And the one-step enzyme detection system
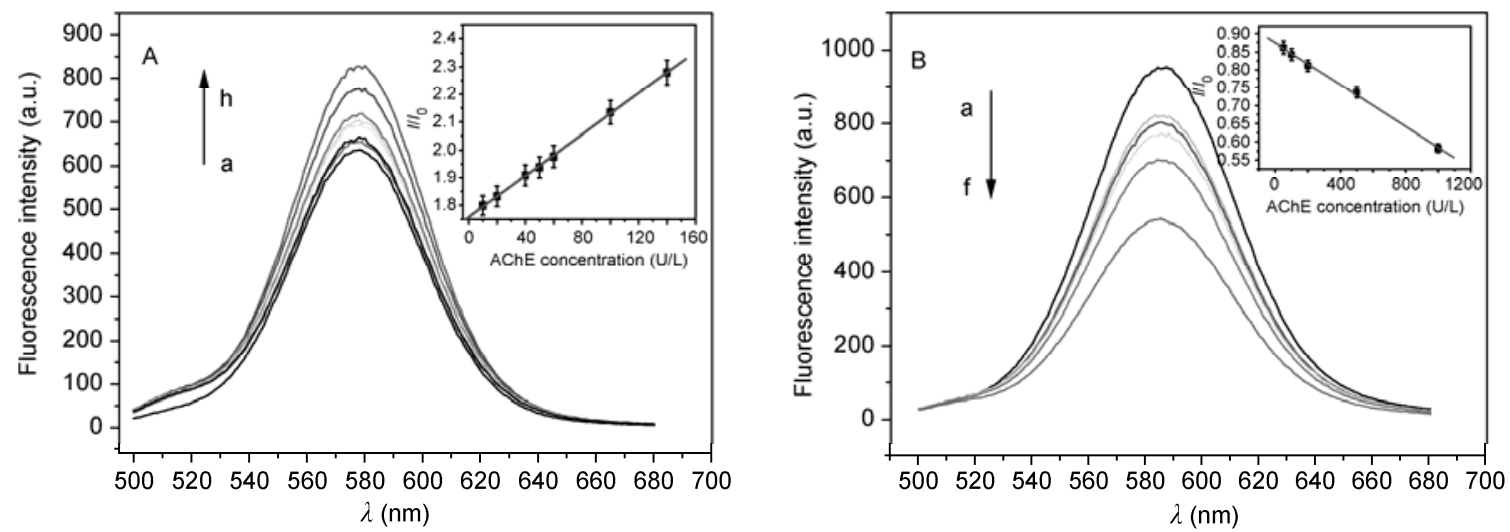

Figure 3 (A) Fluorescence spectra of TGA-QDs in serum with the presence of $0.5 \mathrm{mmol} / \mathrm{L}$ ACh and varying concentrations of AChE. a-h: 0, 10, 20, 40, $50,60,100,140 \mathrm{U} / \mathrm{L}$. The inset displays plots of the relative fluorescence intensity of TGA-QDs versus the concentration of ACh. (B) Fluorescence spectra of MGA-QDs in serum with the presence of $1250 \mathrm{U} / \mathrm{L} \mathrm{ChOx,} 200 \mu \mathrm{mol} / \mathrm{L}$ ACh and varying concentrations of AChE. a-f: 0; 50, 100, 200, 500, 1000 U/L. The inset displays plots of the relative fluorescence intensity of MGA-QDs versus the concentration of ACh.

Table 1 One-step enzyme detection of AChE in serum sample

\begin{tabular}{cccc}
\hline Serum sample & AChE added concentration (U/L) & AChE found concentration (U/L) & Recovery (\%) \\
\hline Sample 1 & 30.0 & 97.2 & 29.2 \\
Sample 2 & 90.0 & 91.0 & 101.1 \\
\hline
\end{tabular}

Table 2 Two-step enzyme detection of AChE in serum sample

\begin{tabular}{|c|c|c|c|}
\hline Serum sample & AChE added concentration (U/L) & AChE found concentration (U/L) & Recovery (\%) \\
\hline Sample 1 & 150.0 & 162.9 & 108.6 \\
\hline Sample 2 & 600.0 & 587.1 & 97.9 \\
\hline
\end{tabular}


makes the detection of AChE cheaper and simpler. Moreover, this work presents a feasible approach for further detecting other kinds of substrate which can be catalyzed to generate acetic acid or $\mathrm{H}_{2} \mathrm{O}_{2}$.

This work was supported by the National Natural Science Foundation of China (81171454 and 61178035).

1 Alivisatos $\mathrm{P}$. The use of nanocrystals in biological detection. Nat Biotechnol, 2004, 22: 47-52

2 Michalet X, Pinaud F F, Bentolila L A, et al. Quantum dots for live cells, in vivo imaging, and diagnostics. Science, 2005, 307: 538-544

3 Gaponik N, Rogach A L. Thiol-capped CdTe nanocrystals: Progress and perspectives of the related research fields. Phys Chem Chem Phys, 2010, 12: 8685-8693

4 Yang Z, Chen C Y, Roy P, et al. Quantum dot-sensitized solar cells incorporating nanomaterials. Chem Comm, 2011, 27: 9561-9571

5 Peng C W, Tian Q, Yang G F, et al. Quantum-dots based simultaneous detection of multiple biomarkers of tumor stromal features to predict clinical outcomes in gastric cancer. Biomaterials, 2012, 33: 5742-5752

6 Pericleous P, Gazouli M, Lyberopoulou A, et al. Quantum dots hold promise for early cancer imaging and detection. Int J Cancer, 2012, 131: 519-528
7 Yuan Y, Zhang J, Liang G L, et al. Rapid fluorescent detection of neurogenin 3 by CdTe quantum dot aggregation. Analyst, 2012, 137: 1775-1778

8 Borisov S M, Wolfbeis O S. Optical biosensors. Chem Rev, 2008, 108: 423-461

9 Bahshi L, Freeman R, Gill R, et al. Optical detection of glucose by means of metal nanoparticles or semiconductor quantum dots. Small, 2009, 5: 676-680

$10 \mathrm{Hu} \mathrm{M}$, Tian J, Lu H T, et al. $\mathrm{H}_{2} \mathrm{O}_{2}$-sensitive quantum dots for the label-free detection of glucose. Talanta, 2010, 82: 997-1002

11 Tang B, Cao L H, Xu K H, et al. A new nanobiosensor for glucose with high sensitivity and selectivity in serum based on fluorescence resonance energy transfer (FRET) between CdTe quantum dots and Au nanoparticles. Chem Eur J, 2008, 14: 3637-3644

12 Yuan J P, Guo W W, Yin J Y, et al. Glutathione-capped CdTe quantum dots for the sensitive detection of glucose. Talanta, 2009, 77: 1858-1863

13 Schebb N H, Fischer D, Hein E M. Fast sample preparation and liquid chromatography-tandem mass spectrometry method for assaying cell lysate acetylcholine. J Chromatogr A, 2008, 1183: 100-107

14 Gaponik N, Talapin D V, Rogach A L, et al. Thiol-capping of CdTe nanocrystals: An alternative to organometallic synthetic routes. J Phys Chem B, 2002, 106: 7177-7185

15 Chen Z Z, Ren X L, Meng X W, et al. Optical detection of choline and acetylcholine based on $\mathrm{H}_{2} \mathrm{O}_{2}$-sensitive quantum dots. Biosens Bioelectron, 2011, 28: 50-55

Open Access This article is distributed under the terms of the Creative Commons Attribution License which permits any use, distribution, and reproduction in any medium, provided the original author(s) and source are credited.

\section{Supporting Information}

Figure S1 Fluorescence spectra of MGA-QDs in the presence of $1 \mathrm{mmol} / \mathrm{L}$ ACh and varying concentrations of AChE. a-e: 0, 40, 100, 400, 1000 U/L. The inset displays plots of the relative fluorescence intensity of MGA-QDs versus the concentration of AChE.

Figure S2 Fluorescence changes of TGA-QDs, the coexistence of TGA-QDs with 1 mmol ACh or 1200 U/L AChE, respectively.

Figure S3 Fluorescence changes of MGA-QDs, the coexistence of MGA-QDs with $200 \mu \mathrm{mol}$ ACh, $1250 \mathrm{U} / \mathrm{L}$ ChOx or 2500 U/L AChE, respectively.

The supporting information is available online at csb.scichina.com and www.springerlink.com. The supporting materials are published as submitted, without typesetting or editing. The responsibility for scientific accuracy and content remains entirely with the authors. 